\title{
PSA-based treatment response criteria in castration-resistant prostate cancer: promises and limitations
}

\author{
Urban Emmenegger, $M$; $^{*}$ Yoo-Joung Ko, MD, MMSc, SM, FRCPC ${ }^{\dagger}$
}

See related article on page 369

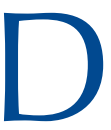

ocetaxel is the first-line cytotoxic treatment of choice for advanced castration-resistant prostate cancer (CRPC) resulting in improved palliation and a modest survival benefit compared to the former standard of care mitoxantrone, as demonstrated by two large randomized trials. ${ }^{1,2}$ The clinical development of superior treatment options and/or second-line therapies has been partly hampered by the lack of validated surrogate markers of treatment benefit. ${ }^{3}$ Treatment response assessment is particularly challenging given that bone is the dominant site of metastasis in CRPC.

Several prostate-specific antigen- (PSA) based response criteria have been studied as potential surrogate markers for survival in CRPC patients treated with docetaxel-based chemotherapy. For instance, $a \geq 30 \%$ PSA decline within 3 months emerged as the optimal surrogate for overall survival in both phase III trials that established docetaxel as the current standard of care. ${ }^{4,5}$ However, the degree of surrogacy of this endpoint varied between these 2 studies and it is not considered as an endpoint for approval of cytotoxic agents by the regulatory agencies. ${ }^{6}$

In this issue of CUAJ, Hanninen and colleagues ${ }^{7}$ present data on another PSA-based endpoint, i.e., PSA half-life (PSAHL), obtained retrospectively from a chart review of 154 patients with metastatic CRPC treated with docetaxel in Alberta between 2000 and 2006. Using the PSAHL determined 84 days following initiation of docetaxel and a cutoff of 70 days, a strong association with survival is demonstrated: the median survival of patients with a PSAHL $<70$ days is 25 months compared to 15 months in patients with a slower rate of PSA decline (PSAHL $\geq 70$ days). Interestingly, the use of PSAHL yielded similar results compared to a $\geq 30 \%$ PSA decline. Both endpoints were more closely correlated with survival when obtained at 3 months and 84 days, respectively, than during earlier time points. Furthermore, the application of these criteria allows the stratifying of CRPC patients with similar survival.

Although the prospective study of $\geq 30 \%$ PSA decline and PSAHL might be considered a reasonable next step, one can question whether this alone will provide the treating physician with a tool to predict who will not benefit from docetaxel chemotherapy, and hence could be spared the docetaxel-related side effects and be potentially recruited for clinical trials exploring alternative therapies. In fact, the use of PSA as a marker of response in docetaxel-treated CRPC has several limitations. First, in vitro, docetaxel has been shown to down-regulate androgen receptor expression and PSA expression/secretion, ${ }^{8}$ which could explain the discrepancy commonly seen in clinical trials between the rate of PSA and measurable disease responses. ${ }^{1,2}$ Second, CRPC is a heterogeneous disease (intra- and inter-individually) with respect to PSA expression and to chemosensitivity. ${ }^{9}$ Thus, the differential treatment impact on CRPC cells with distinct levels of PSA expression may complicate the interpretation of PSA data. Third, up to $20 \%$ of patients can experience PSA flares in the first weeks of docetaxel therapy despite subsequent response, which challenges the use of PSA-based criteria as an early marker of treatment benefit. ${ }^{6}$ Finally, the interpretation of PSA data will be even more obscured with the increasing use of molecularly targeted agents, some of which result in dissociated antitumour/PSA effects, as is the case for sorafenib. ${ }^{10}$

The authors acknowledge that the retrospective nature of the study is a major limitation. Although the investigators have defined the inclusion criteria of the cohort, other important data, such as dose and schedule of chemotherapy, were not detailed. The study would have been strengthened if the authors were able to similarly analyze the PSA data from the two large randomized phase III docetaxel studies, or if they were able to validate their findings in a separate cohort of patients.

Despite these limitations, PSA-based criteria will undoubtedly continue to have an important role in treatment assessment of men with CRPC in the foreseeable future, possibly as part of combinatorial endpoints that might also involve palliative criteria, novel biological surrogate markers and functional imaging. ${ }^{6}$

*Division of Medical Oncology, Department of Medicine, Sunnybrook Odette Cancer Centre, and Molecular and Cellular Biology Research, Sunnybrook Research Institute, Sunnybrook Health Sciences Centre, University of Toronto, Toronto, ON; ' ${ }^{\circ}$ ivision of Medical Oncology, Department of Medicine, Sunnybrook Odette Cancer Centre, Sunnybrook Health Sciences Centre, University of Toronto, Toronto, ON 
Competing interests: Dr. Emmenegger and Dr. Ko have received speaker fees from Novartis Canada.

This paper has been peer-reviewed.

\section{References}

1. Tannock IF, de Wit R, Berry WR, et al. Docetaxel plus prednisone or mitoxantrone plus prednisone for advanced prostate cancer. N Engl J Med 2004;351:1502-12.

2. Petrylak DP, Tangen CM, Hussain MH, et al. Docetaxel and estramustine compared with mitoxantrone and prednisone for advanced refractory prostate cancer. N Engl J Med 2004;351:1513-20.

3. Scher HI, Halabi S, Tannock I, et al. Design and end points of clinical trials for patients with progressive prostate cancer and castrate levels of testosterone: recommendations of the Prostate Cancer Clinical Trials Working Group. J Clin Oncol 2008;26:1148-59.

4. Petrylak DP, Ankerst DP, Jiang CS, et al. Evaluation of prostate-specific antigen declines for surrogacy in patients treated on SWOG 99-16. J Natl Cancer Inst 2006;98:516-21.
5. Armstrong AJ, Garrett-Mayer E, Ou Yang YC, et al. Prostate-specific antigen and pain surrogacy analysis in metastatic hormone-refractory prostate cancer. J Clin Oncol 2007;25:3965-70.

6. Armstrong AJ, Febbo PG. Using surrogate biomarkers to predict clinical benefit in men with castrationresistant prostate cancer: an update and review of the literature. Oncologist 2009;14:816-27.

7. Hanninen $M$, Venner $P$, North $S$. A rapid PSA half-life following docetaxel chemotherapy is associated with improved survival in hormone refractory prostate cancer. Can Urol Assoc I 2009;3:369-74.

8. Kuroda K, Liu H, Kim S, et al. Docetaxel down-regulates the expression of androgen receptor and prostate-specific antigen but not prostate-specific membrane antigen in prostate cancer cell lines: implications for PSA surrogacy. Prostate 2009;69:1579-85.

9. Shah RB, Mehra R, Chinnaiyan AM, et al. Androgen-independent prostate cancer is a heterogeneous group of diseases: lessons from a rapid autopsy program. Cancer Res 2004;64:9209-16.

10. Chi KN, Ellard SL, Hotte SJ, et al. A phase II study of sorafenib in patients with chemo-naive castrationresistant prostate cancer. Ann Oncol 2008;19:746-51.

Correspondence: Dr. Urban Emmenegger, Sunnybrook Health Sciences Centre, 2075 Bayview Avenue, Toronto, Ontario M4N3M5; fax: 416-480-6002; urban.emmenegger@sunnybrook.ca

\section{Correction}

In an original research article from the June 2008 issue of CUAJ entitled "Vardenafil demonstrates first-dose success and reliability of penetration and maintenance of erection in men with erectile dysfunction-RELY-II,"1 Figure 2 was incorrect. The correct figure is published here.

\section{Reference}

1. Valiquette L, Montorsi F, Auerbach S, for the Vardenafil Study Group. Vardenafil demonstrates firstdose success and reliability of penetration and maintenance of erection in men with erectile dysfunction-RELY-II. Can Urol Assoc J 2008;2:187-95.

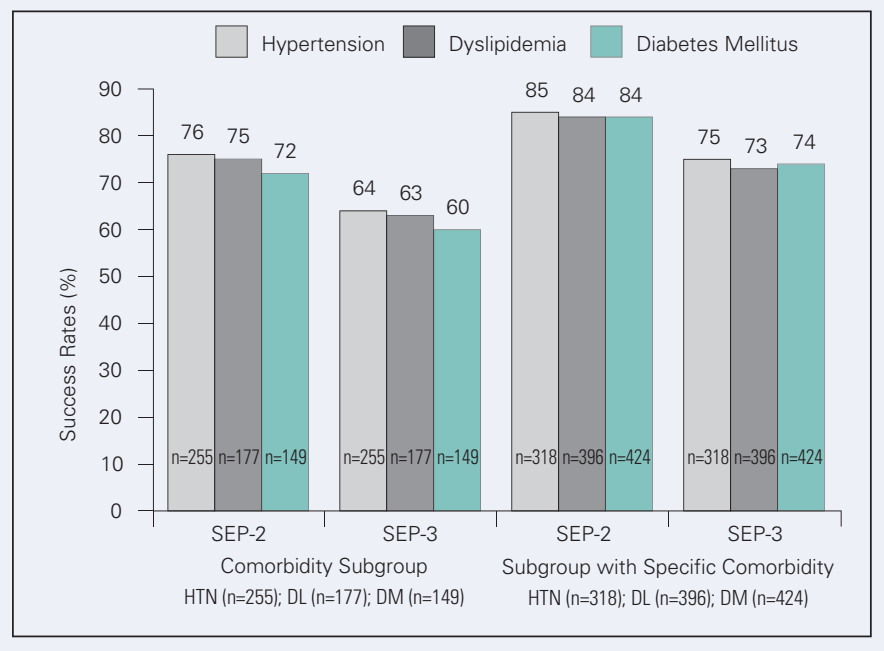

